\title{
Cutting Parameters Optimisation in Milling: Expert Machinist Knowledge versus Soft Computing Methods
}

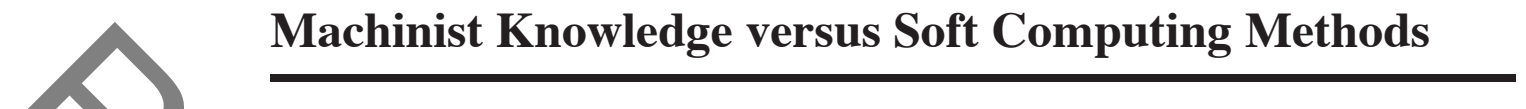

\section{J.V. Abellan-Nebot}

Department of Industrial Systems Engineering and Design, School of Technology and Experimental Sciences, Universitat Jaume I, 12071 Castellon, Spain Fax: +34964728170_E-mail: abellan@esid.uji.es

\section{Abstract}

In traditional machining operations, cutting parameters are usually selected prior to machining according to machining handbooks and user's experience. However, this method tends to be conservative and sub-optimal since part accuracy and non machining failures prevail over machining process efficiency. In this paper, a comparison between traditional cutting parameter optimisation by an expert machinist and an experimental optimisation procedure based on Soft Computing methods is conducted. The optimisation procedure presented is composed of two steps: (1) modelling the process variables of interest and (2) optimising a multi-objective function to reach a trade-off among production rate, cutting costs and part accuracy. The first step applies an Adaptive Neuro-fuzzy Inference System (ANFIS) to model the process by extracting rules from experimental data. The second step applies Genetic Algorithms to optimise the multi-objective function which is defined by an overall desirability function approach. The proposed methodology increased the machining performance in $6.1 \%$ and improved the understanding of the machining operation through the ANFIS models.

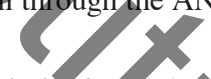

Keywords: Machining, Parameters Optimisation, Adaptive Neuro-Fuzzy Inference Systems, Soft Computing, Genetic Algorithms, Multi-Objective Functions, Expert Machinist, Desirability Functions.

Reference to this paper should be made as follows:

Biographical Notes: José V. Abellán-Nebot is assistant professor in the Department of Industrial Systems Engineering and Design at Jaume I University. He received the M.Sc. degree in manufacturing engineering in 2003. In 2004, he joined the Department as a research assistant. Since 2007, he lecturers Computer Integrated Manufacturing, Computer Aided Manufacturing and Manufacturing Technologies. He was a visiting scholar at the Centre for Innovation in Design and Technology located at Monterrey Institute of Technology (México) in 2005 and at the Engineering Research Center for Reconfigurable Manufacturing Systems (ERC-RMS) at University of Michigan in 2007. He is author of more than fifteen conference proceedings and several research studies published at international journals. His research interests include issues related to intelligent machining systems, stream of variation in machining systems and manufacturing and collaborative engineering. 


\section{Introduction}

Metal cutting is one of the important and widely used manufacturing processes in engineering industries. The study of metal cutting focuses, among others, on the features of tools, input work materials, and machine parameter settings influencing machining process efficiency and output quality characteristics. A significant improvement in machining process efficiency may be obtained by process parameter optimisation that identifies and determines the regions of critical process control factors leading to desired outputs or responses with lower manufacturing cost (Mukherjee and Ray, 2006). However, in traditional Computer Numerical Control (CNC) systems machining parameters are usually selected prior to machining according to machining handbooks or user's experience, which is a conservative and sub-optimal procedure to assure part quality specifications without machining failure.

In order to overcome the limitations of traditional cutting parameter selection, experimental process parameter optimisation procedures should be applied in industry. These procedures are composed of two steps: (1) modelling of process variables relationship through experimentation, and (2) determination of optimal or near-optimal cutting conditions through optimisation algorithms. First step deals with representing the machining process through mathematical models required for a later formulation of the process objective function. In the literature, several modelling techniques have been implemented mainly based on statistical regressions (Cus and Balic, 2003), artificial neural networks (Liu and Wang, 1999a; Chiang et al, 1995; Liu et al., 1999b; Zuperl and Cus, 2003; Chien and Chou, 2001) and fuzzy set theory (Ip, Lau and Chan, 2003). Second step provides optimal or near-optimal solution(s) to the oyerall optimisation problem formulated through the previous mathematical models. In the literature, the main optimisation tools and techniques applied are based on Taguchi method (Ghani, Choudhury and Hassan, 2004; Zhang, Chen and Kirby, 2007), response surface design (Suresh, Rao and Deshmukh, 2002), genetic algorithms (Liu, Zuo and Wang, 1999b; Suresh, Rao and Deshmukh, 2002; Cus and Balic, 2003; Chien and Chou, 2001) and simulated annealing (Juan, Yu and Lee, 2003).

Cutting parameter optimisation in machining has been intensively studied in the literature as it can be stated in a recent review (Mukherjee and Ray, 2006) Liu and Wang (1999a) proposed an adaptive control system based on two neural network models, a BackPropagation Neural Networks (BP NN) and an Augmented Lagrange Multiplier Neural Network (ALM NN). The BP NN was used for modelling the state of the milling system, using as a single input the feed parameter and sensing the cutting forces on-line. The ALM $\mathrm{NN}$ was used for maximising the material removal rate which it was carried out adjusting the feed rate. Chiang et al. (1995) presented a similar work for end-milling operations, but surface roughness was also considered as constraint. Both research works were based on theoretical formulas for training the neural networks and both applied an ALMNN for optimisation. Liu, Zuo and Wang (1999b) also extended his previous work with a new optimisation procedure based on a Genetic Algorithm (GA). Ghani, Choudhury and Hassan (2004) optimised cutting parameters using a Taguchi's Design of Experiments in end milling operations. With a minimum number of trials compared with other approaches such as a full factorial design, the methodology presented reveals the most significant factors and interactions during cutting process which leads to choose the optimal conditions. A very similar methodology was described by Zhang, Chen and Kirby (2007). However, both methodologies do not permit to evaluate quadratic or non-linear relations between factors,

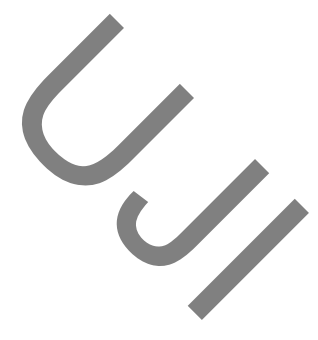


and the analysis is restricted to the levels analysed in each factor. A more generic approach although more costly in experiments is based on Response Surface Model optimisation (RSMO). Suresh, Rao and Deshmukh (2002) used the Response Surface Methodology (RSM) for modelling the surface roughness as a first and second-order mathematical model. The surface roughness optimisation was carried out through GA which are time consuming and are more appropriate for optimising non-linear functions. Cus and Balic (2003) also applied GA for optimising a multi-objective function based on minimum time necessary for manufacturing, minimum unit cost and minimum surface roughness. All the process models applied in their research were empirical formulaes from machining handbooks and they were adjusted through regressions. More complex models have been also applied for surface roughness and tool wear modelling to optimise off-line cutting parameters. Zuperl and Cus (2003) also applied and compared feed-forward and radial basis neural networks forlearning a multi-objective function similar to the one presented by Cus and Balic (2003). Choosing the radial basis networks due to their fast learning ability and reliability, they applied a large-scale optimisation algorithm to obtain the optimal cutting parameters. Chien and Chou (2001) applied neural networks for modelling surface roughness, cutting forces and cutting-tool life and applied a GA to find optimum cutting conditions for maximising the material removal rate under the constraints of the expected surface roughness and tool life. Ip, Lau and Chan (2003) applied fuzzy sets to optimise the material removal rate in the manufacturing of seulptured surfaces and they demonstrated a material removal rate increase of $41 \%$ in comparison with conventional constant feedrate. Juan, Yu and Lee (2003) applied polynomial networks to model a roughing milling operation, and the production cost was minimised using a simulated annealing method. Table 1 summaries recent research works related to cutting parameter optimisation.

Table 1 Literature review on cutting parameter optimisation.

\begin{tabular}{lcccc} 
Reference & Modelling & Optimisation & Output \\
Approach & Approach & Optimised & Process \\
\hline (Liu and Wang, 1999a) & Feed-forward NN & ALM NN & MRR & M \\
(Chiang et al., 1995) & Feed-forward NN & ALM NN & MRR & EM \\
(Liu et al., 1999b) & Feed-forward NN & GA & MRR & M \\
(Ghani et al., 2004) & - & CF, Ra & EM \\
(Zhang et al., 2007) & - & TA & Ra & EM \\
(Suresh et al., 2002) & Response Surface & GA & Ra & T \\
(Cus and Balic, 2003) & Statistical Regressions & GA & Unit cost, Ra & T \\
& & & Time & \\
(Zuperl and Cus, 2003) & Feed-forward and & LSO & MRR, Tl & T \\
(Chien and Chou, 2001) & Radial Basis NN & & Ra & T \\
(Ip et al., 2003) & Feed-forward NN & GA & MRR & M \\
(Juan et al., 2003) & Fuzzy Sets & - & MRR & M \\
\hline
\end{tabular}

MRR: Material Removal Rate; TI: Tool-life; Ra: Surface Roughness; CF: Cutting Force;

GA: Genetic Algorithm; TA: Taguchi Approach; ALM NN: Augmented Lagrange Multiplier;

LSO: Large Scale Optimisation; SA: Simulated Annealing

T: Turning; EM: End Milling; M: Milling;

In this paper, the traditional optimisation procedure based on machinist's expertise is compared with an experimental cutting optimisation procedure based on Soft Computing methods. The Soft Computing methods applied to model the process is the Adaptive NeuroFuzzy Inference System (ANFIS), which describes the process knowledge in a form of If- 
Then rules. Unlike other methods presented in the literature such as Neural Networks (NN), Fuzzy, Response Surface (see Table 1 1 , ANFIS models let understand the process through simple rules extracted from experimental data, so the expert machinist is able to learn new knowledge from a particular machining operation and confirm well-known machining practices/rules. The comparison is conducted in a finishing face-milling operation with Cubic Boron Nitride (CBN) cutting tools, which are modern and expensive cutting tools. The cutting parameter optimisation deals with a multi-objective function where surface roughness, tool life, material removal rate and quality loss functions are considered through a desirability function approach. The final optimisation algorithm is based on another soft computing method called Genetic Algorithm (GA).

\section{Machining Process Description}

The machining process studied in this paper is presented in Figure1, and it consists of a face-milling operation on workpieces of hardened AISI D3 steel $(60 \mathrm{HRc})$ with dimensions $250 \times 250 \mathrm{~mm}$. The experiments were conducted on a CNC machining centre suited for mould and die manufacturing, and the cutting tool used was a face milling tool with Cubic Boron Nitride (CBN) inserts, In order to generate a good surface finish and avoid run-out problems, a single insert was mounted on a tool body with an effective diameter of $40 \mathrm{~mm}$.

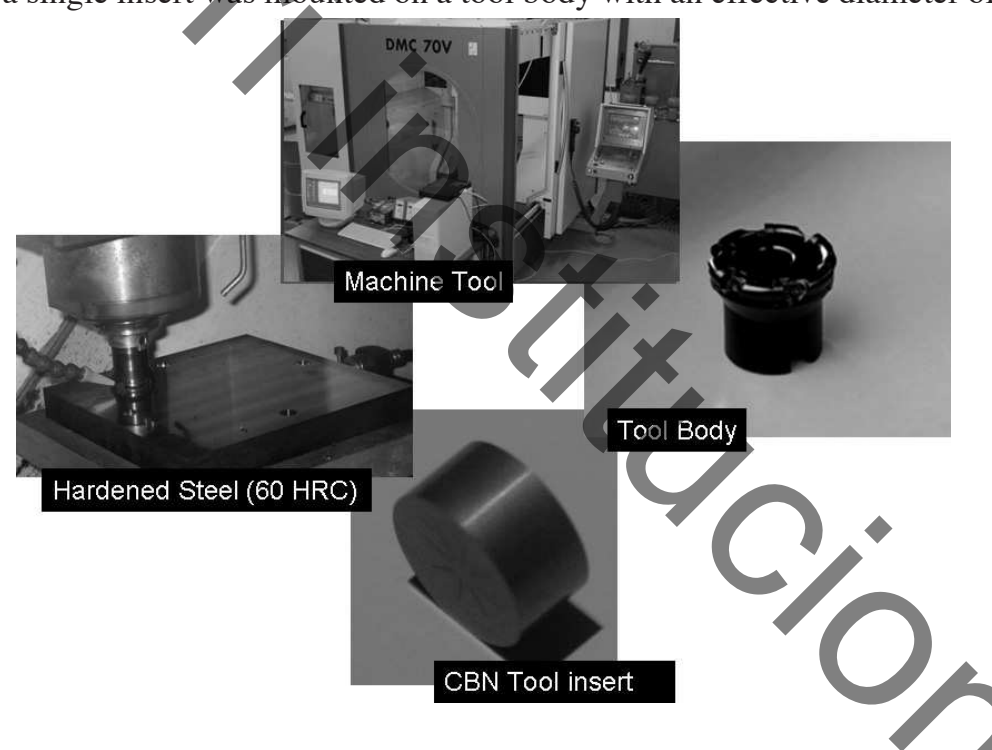

Figure 1 Machining process analysed: face-milling operation on workpieces of hardened-AISI D3 steel $(60 \mathrm{HRc})$ with dimensions $250 \times 250 \mathrm{~mm}$ and Cubic Boron Nitride $(\mathrm{CBN})$ cutting inserts.

\section{Definition of the Optimisation Problem}

\subsection{Objective Functions}

Typically, three objective functions are considered in a cutting parameters optimisation problem: material removal rate, surface roughness and cutting-tool life. Material Removal Rate $(M R R)$ is a measurement of productivity, and it can be expressed by equation (10, 
where $V_{c}, f_{z}$ and $a_{p}$ are the cutting speed, feed rate per tooth and depth of cut respectively. Most of the cutting parameter optimisation procedures in roughing operations try to maximise $M R R$ constrained to cutting forces (Liu and Wang, 1999a). Surface roughness ( $R a$ ) is the most important criterion for the assessment of the surface quality, and it is usually calculated empirically through experiments. Surface roughness can be described as an empirical relationship among the cutting parameters $V_{c}, f_{z}, a_{p}$, and it is commonly minimised for high quality machining operations (Zhang, Chen and Kirby, 2007; Suresh, Rao and Deshmukh, 2002). Cutting-tool life $(T)$ is the other important criterion for cutting parameters selection, since several costs such as cutting-tool replacement cost and cutting-tool cost are directly related with cutting-tool life. The tool life can be also described as an empirical relationship among the cutting parameters $V_{c}, f_{z}, a_{p}$, and it is usually fixed to find a trade-off between cutting-tool/replacement costs and production rate (Cus and Balic, 2003; Zuperl and Cus, 2003). In addition to these three objective functions, the Taguchi's loss quality function $(W$ ) can be another important objective function for finishing operations since the surface roughness variability along part surface impacts on the final part quality. Considering a desired $R a$ value, the quality loss function is usually applied to estimate the cost of manufacturing with a quality variation. As $R a, W$ is commonly minimised for high quality machining operations. Therefore, the four objective functions which have to be taken into consideration for an optimal çutting parameter selection can be listed as follows:

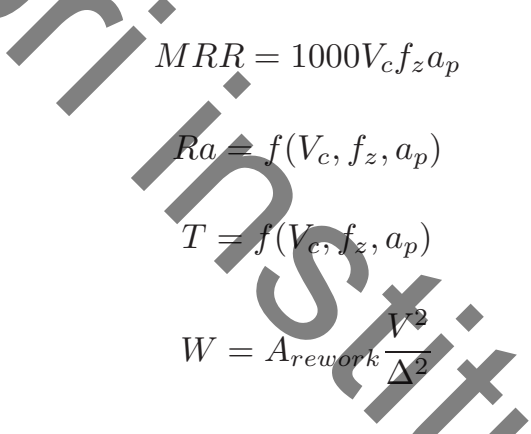

where $\Delta=R a_{\max }-R a_{\text {target }} . R a_{\max }$ and $R a_{\text {target }}$ define the maximum Ra by part specifications and the Ra desired respectively. $V^{2}$ defines the mean squared deviation as $V^{2}=\left(\left(R a_{\text {target }}-y_{1}\right)^{2}++\left(R a_{\text {target }}-y_{n}\right)^{2}\right) / n$, with $n$ the number of samples and $A_{\text {rework }}$ is the part cost if the part is outside specifications. In several research works (Suresh, Rao and Deshmukh, 2002; Cus and Balic, 2003), the surface roughness and cutting tool life functions have been described by well-known empirical equations as follows:

$$
\begin{aligned}
& R_{a}=k V^{x_{1}} f_{z}^{x_{2}} a_{p}^{x_{3}} \\
& T=\frac{K_{T}}{V^{\alpha_{1}} f_{z}^{\alpha_{2}} a_{p}^{\alpha_{3}}}
\end{aligned}
$$

where $k, x_{1}, x_{2}, x_{3}, K_{T}, \alpha_{1}, \alpha_{2}, \alpha_{3}$ are empirical coefficients. However, for high quality machining operations with CBN cutting tools, both equations may not provide a good estimation. The main reason is that additional mechanisms such as vibrations, engagement of the cutting tool or built up edge influence the surface roughness generation when machining at very low feed speeds (Siller et al., 2008) . Furthermore, CBN tools have a different wear process than traditional cutting-tools such as high speed steels, so equation (6) may not be directly applied (Trent and Wright, 2000). 


\subsection{Multi-Objective Function}

The optimisation problem for the case study is defined as the optimisation of a multiobjective function which is composed of the objective functions defined by Equations 11 , 2, 3 and (4). Due to these objective functions are conflicting and incomparable, the multiobjective function is defined using the desirability function approach. The desirability function approach is based on the idea that the optimal performance of a process that has multiple performance characteristics is reached when the process operates under the most desirable performance values (NIST/SEMATECH, 2006). For each objective function $Y_{i}(x)$, a desirability function $d_{i}\left(Y_{i}\right)$ assigns numbers between 0 and 1 to the possible values of $Y_{i}$, with $d_{i}\left(Y_{i}\right)=0$ representing a completely undesirable value of $Y_{i}$ and $d_{i}\left(Y_{i}\right)=1$ repreenting a completely desirable or ideal objective value. Depending on whether a particular objective function $Y_{i}$ is to be maximised or minimised, different desirability functions $d_{i}\left(Y_{i}\right)$ can be used. A useful class of desirability functions was proposed by Derringer and Suich (1980). Let $E_{i}$ and $U_{i}$ be the lower and upper values of the objective function respectively, with $L_{i}<\tau_{i}$, and let $T_{i}$ be the desired value for the objective function. Then, if an objective function $Y_{i}(x)$ is to be maximised, the individual desirability function is defined as

$$
d_{i}\left(Y_{i}\right)=\left\{\begin{array}{clc}
0 & \text { If } & Y_{i}(x)<L_{i} \\
\left(\frac{Y_{i}-L_{i}}{T_{i}-L_{i}}\right)^{w} & \text { If } L_{i} \leqslant Y_{i}(x) \leqslant T_{i} \\
\frac{1}{1} & \text { If } & Y_{i}(x)>T_{i}
\end{array}\right.
$$

where the exponent $w$ is a weighting factor which determines how important it is to hit the target value. For $w=1$, the desirability function increases linearly towards $T_{i}$; for $w<1$, the function is convex and there is less emphasis on the target; and for $w>1$, the function is concave and there is more emphasis on the target. If one wants to minimise an objective function instead, the individual desirability is defined as

$$
d_{i}\left(Y_{i}\right)=\left\{\begin{array}{cl}
1 & \text { If } \quad Y_{i}(x)<T_{i} \\
\left(\frac{Y_{i}-U_{i}}{T_{i}-U_{i}}\right)^{w} & \text { If } T_{i} \leqslant Y_{i}(x) \leqslant U_{i} \\
0 & \text { If } \quad Y_{i}(x)>U_{i}
\end{array}\right.
$$

Figure 2 shows the individual desirability functions according to different $w$ values. The individual desirability functions are combined to defined the multi-objective function, called the overall desirability of the multi-objective function. This measure of composite desirability is the weighted geometric mean of the individual desirabilities for the objective functions. The optimal solution (optimal operating conditions) can then be determmed by maximising the composite desirability. The individual desirabilities are weighted by importance factors $I_{i}$. Therefore, the multi-objective function or the overall desirability function to optimise is defined as:

$$
D=\left(d_{1}\left(Y_{1}\right)^{I_{1}} \times d_{2}\left(Y_{2}\right)^{I_{2}} \times \cdots \times d_{k}\left(Y_{k}\right)^{I_{k}}\right)^{1 /\left(I_{1}+I_{2}+\cdots+I_{k}\right)}
$$

with $k$ denoting the number of objective functions and $I_{i}$ is the importance for the objective function $i(i=1,2, \cdots, k)$. 

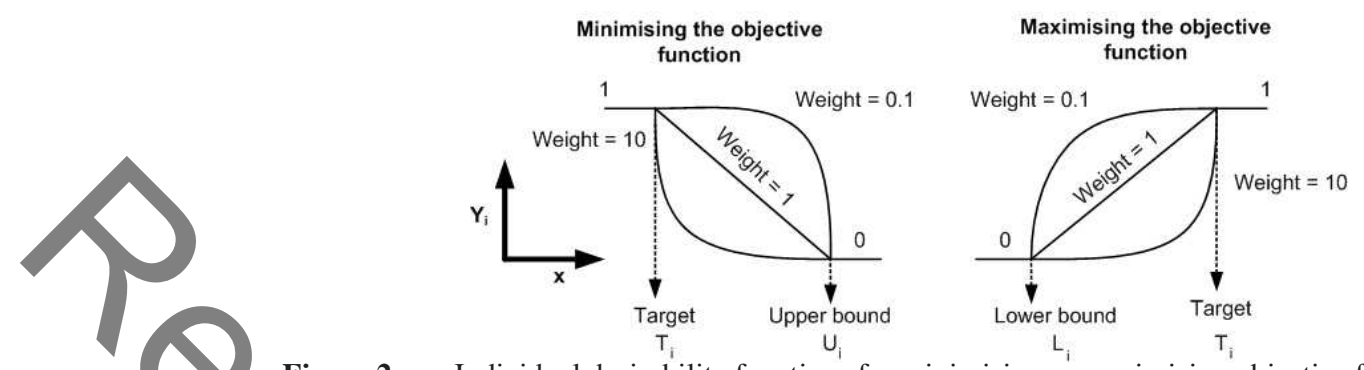

Figure 2 Individual desirability functions for minimising or maximising objective functions. The weighting factor determines how the desirability function increases/decreases.

3.3 Constraints

Due to the limitations on the cutting process, manufacturers limit the operation range of cutting parameters to avoid premature cutting-tool failures. Therefore, the cutting parameter selection according to manufacturer specifications is constrained to:

$$
V_{\min } \leqslant V_{c} \leqslant V_{\max } \quad f_{\min } \leqslant f_{z} \leqslant f_{\max } \quad a_{p} \leqslant a_{\max }
$$

Surface roughness and quality loss specification are also considered as constraints that can be expressed as

$$
R a \leqslant R a_{\text {spec }} \quad W \leqslant W_{\max }
$$

In addition, cutting power and force limitations are usual constraints, but they are more commonly applied in roughing operation

\subsection{Summary of Optimisation Problem and Numerical Coefficients}

The weights and the individual desirability coefficients for each objective function were chosen according to machining process characteristics. First the weights were defined considering how the objective function increases or decreases as the ideal value is not matched. In order to simplify the desirability functions, the weighting factor at eachdesirability function was set to 1 , so all desirability functions were defined as linear functions where the desirability increases linearly towards the target value. Secondly, the coefficients of importance for each objective function were set according to machining requirements. For the case study, it was assumed that the machining process requires as first objective a high production rate (high $M R R$ ), a low cutting-tool costs (high $T$ ) as a second objective, and as a third and less important objective a high part quality (low $R a$ and low W). In order to determine the overall desirability function (the multi-objective function), a numerical approximation of the relative importance among individual objective functions is required. Although this estimation is subjective, it can be an easy and fast way to approximate the cutting parameter selection to the near-optimal one. For a more accurate definition of multiobjective functions it is required the definition of all machining costs, cutting-tools, labour costs, product sale price, machine-tool operation cost, etc., which can be in some cases difficult to know. For the case study, the coefficient of importance for each desirability function were set to $4,1,0.75,0.75$ for $M R R, T, R a$ and $W$ respectively. Considering the maximum and minimum value of each objective function obtained analytically, the desirability functions were defined as follows. 
- $M R R$ desirability function

$$
d_{1}(M R R)=\frac{M R R-390}{2387-390}
$$

$M R R_{\text {target }}=2387 \mathrm{~mm}^{3} / \mathrm{min} . M R R_{\text {minimum }}=390 \mathrm{~mm}^{3} / \mathrm{min}$. Importance factor $I_{1}=4$.

- Desirability function of $R a$ deviation objective function

$$
d_{2}(W)=d_{2}\left(V^{2}\right)=\frac{V^{2}-0.013}{0.0001-0.013}
$$

$V_{\text {target }}^{2}=0.0001 \mu \mathrm{m}^{2} . V_{\text {maximum }}^{2}=0.013 \mu \mathrm{m}^{2}$. Importance factor $I_{2}=0.75$. Note that the desirability function of quality loss $W$ for surface roughness can be defined by the surface roughness deviation $V^{2}$ since Equation (4) relates $W$ with $V^{2}$ by a constant coefficient of $A_{\text {rework }} / \Delta^{2}$.

- Ra desirability function
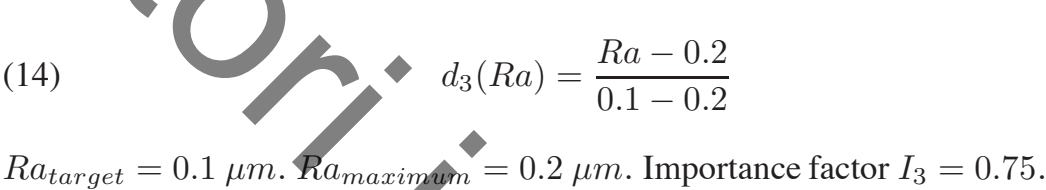

- Cutting-tool life desirability function

$$
T_{\text {target }}=46.7 \mathrm{~min} . T_{\text {minimum }}=6 \text { min. Importance factor } I_{4}=1 .
$$

The multi-objective function or the overall desirability function to be optimised is:

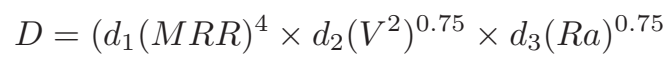
constrained to:

(17) $100 \mathrm{~m} / \mathrm{min} \leqslant V_{c} \leqslant 200 \mathrm{~m} / \mathrm{min}$

$$
a_{p}=4 m m \quad R a \leqslant 0.2 \mu m
$$

$$
0.04 \mathrm{~mm} / \mathrm{rev} \leqslant f_{z} \leqslant 0.12 \mathrm{~mm} / \mathrm{rev}
$$

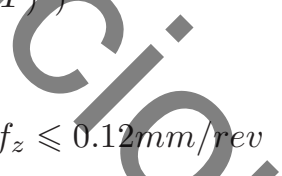

$V^{2} \leqslant 0.013 \mu m$

\section{Parameter Optimisation based on an Expert Machinist}

\subsection{Methodology}

Cutting tool parameters are traditionally chosen according to handbooks, cutting-tool data catalogs and machinist's experience. For a given cutting-tool and workpiece material, a range of possible cutting-parameters are provided by the cutting-tool supplier, and the machinist chooses the parameters within the admissible operation ranges using some wellknown practices in shop-floor. Some of these practices are: 


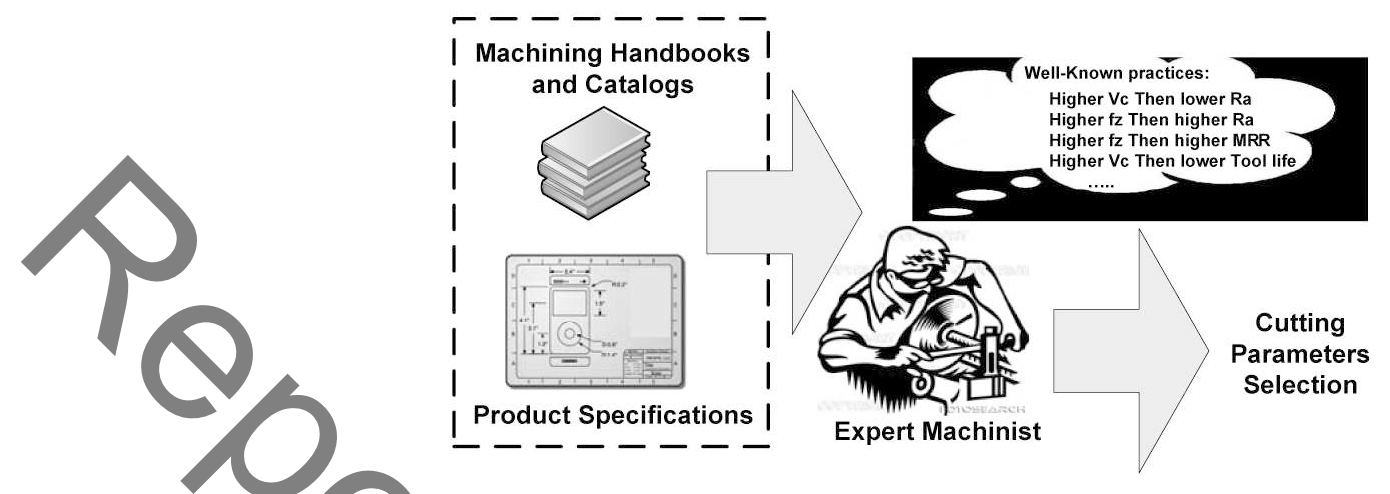

Figure 3 Parameter optimisation based on an expert machinist knowledge. Knowing product specifications and using handbooks and catalogs, the expert selects the optimal cutting parameters.

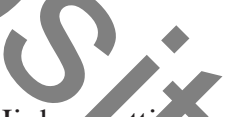

- Higher cutting speeds increase surface roughness quality but decrease cutting tool life.

- Higher cutting speeds decrease cutting tool life.

- Higher feed rates increase productivity as it is increased material removal rate.

- Higher feed rates decrease surface roughness quality.

- Higher feed rates decrease cutting-tool life.

- Higher axial depths of cut increase productivity.

- Higher axial depths of cut decrease cutting-toollife.

- Very low axial depths of cut burn the workpiece surface and generate a low surface roughness quality decreasing cutting-tool life.

According to the final goal of the machining process, the machinist selects the best cutting-tool parameters combination. For example, if the only important constraint was a high cutting tool life, the machinist would select a low cutting speed, low feed rate and lowmedium axial depth. Figure 3 describes the typical optimisation proeess based on machinist expertise.

\subsection{Optimisation Results}

As it was explained above for the case study (section 3.4), the MRR was considered the most important objective function with an importance coefficient of 4 whereas less important are tool-life, surface roughness and loss quality with importance coefficients of $1,0.75$ and 0.75 respectively. Considering only a maximum MRR value as an objective function, the machinist would fix the maximum cutting speed and feed rate. However, although desirability functions of $\mathrm{Ra}$ and $\mathrm{W}$ are less important, the machining process would require low cutting speeds and low-medium feed rates in order to maximise them. In addition, the tool life objective function which is also less important than MRR would require low cutting speeds and low-medium feed rates. As the importance coefficient of the MRR objective 
function is four times more important than the others, and a medium feed rate could increase the output of the objective functions surface roughness, loss quality and tool-life, the expert machinist decided to fix the near-optimal cutting parameters at $V_{c}=200 \mathrm{~m} / \mathrm{min}$, $f_{z}=0.08 \mathrm{~mm}$.

An experimentation was conducted in order to check the overall desirability function at these cutting conditions, which are the near-optimal according to expert machinist's reasoning. The experimental results showed a cutting tool life of $T=10.8 \mathrm{~min}$, an average surface roughness deviation of $V^{2}=1.6 \times 10^{-3} \mu \mathrm{m}^{2}$, a surface roughness of $R a=0.135$ tum and a $M R R=1592 \mathrm{~mm}^{3} / \mathrm{min}$. The overall desirability was 0.505 .

\section{Parameter Optimisation based on AI \\ 5.1 Methodology}

The experimentalcutting parameters optimisation methodology based on soft computing methods is shown in Figure 4. The first step of the methodology consists of an experimentation based on a Design of Experiments in order to obtain the performance of the process in the cutting parameter region to be studied. After experimentation, the experimental data is used to model the process through an Adaptive Neuro-fuzzy Inference Systems (ANFIS), a soft computing technique which can describe a process by a model using rules extracted from experimental data. This technique lets extract knowledge from the process in a If-Then form which can be used for understanding the process. An ANFIS model was developed for each variable of interest: $R d, M R R, T$ and $V^{2}$. With these models, each individual desirability function could be estimated by an ANFIS model, and the overall desirability function could be defined by the individual desirability functions according to equation (16). Finally, the cutting parameter optimisation was carried out through another soft computing technique called Genetic Algorithms (GA) considering product and process specifications.

Next subsections briefly review both soft computing methods: Adaptive Neural Fuzzy Inference Systems and Genetic Algorithms.

\subsubsection{Adaptive Neural Fuzzy Inference Systems}

Adaptive-Network-based Fuzzy Inference System (ANFIS) is a method for the fuzzy modelling procedure to learn information about a data set, in order to compute the membership function parameters that best allow the associated fuzzy inference system to track the given input/output data. This learning method works similarly to that of neural networks. Using a given input/output data set, the membership function parameters of the fuzzy inference system are tuned using either a backpropagation algorithm alone, or in combination with a least squares type of method. The parameters associated with the membership functions will change through the learning process. In general, this type of modelling works well if the training data presented for training the membership function parameters is fully representative of the features of the data that the trained Fuzzy Inference System (FIS) is intended to model.

ANFIS is functionally equal to the Takagi and Sugeno's inference mechanism. This type of inference system can be represented by an adaptable and hybrid neural network

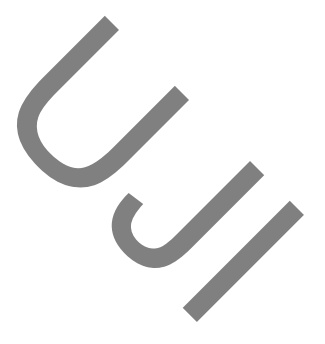




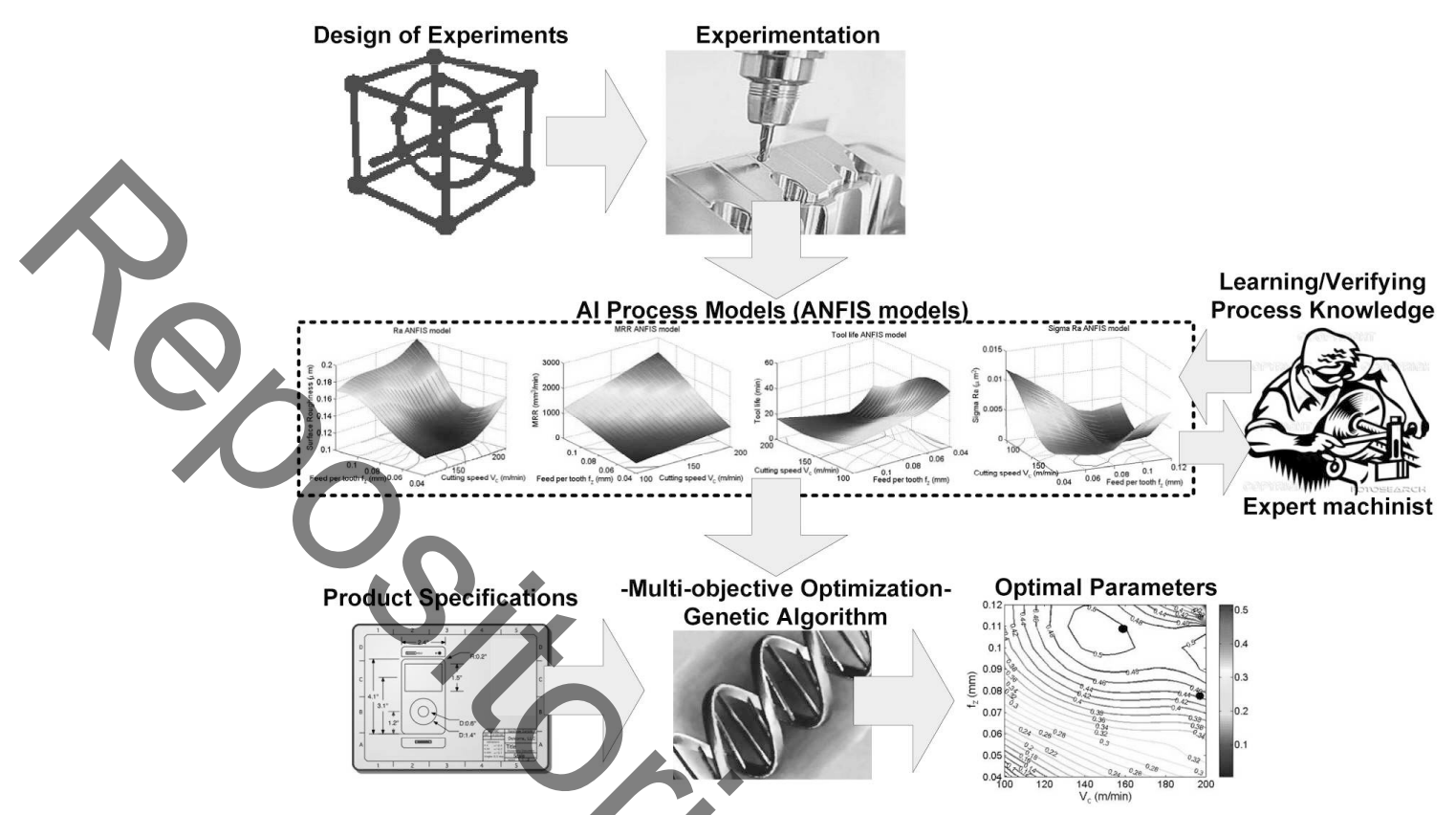

Figure 4 Parameter optimisation based on Soft Computing methods. ANFIS models are built after conducting a Design of Experiments, and the If-Then rules extracted are verified by an expert machinist. The ANFIS models are used to build the overall desirability function according to cutting speeds and feed rates. A Genetic Algorithm is applied to find the optimal parameters considering product and process specifications.

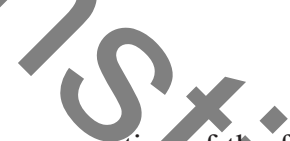

with 5 layers. Each layer represents an operation of the fuzzy inference mechanism. Assuming an ANFIS structure defined with two inputs $x$ and $y$ and one output $z$ (Figure 5), the characteristics of each layer can be described as follows (Jang, 1993):

1. Layer 1, the fuzzy layer: Every node $i$ in this layer is an adaptable node with a node function

$$
O_{i}^{1}=\mu_{A_{i}}(x)
$$

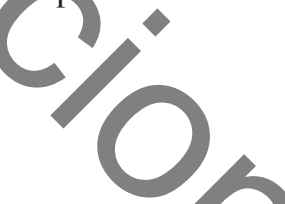

where $x$ is the input to node $i$, and $A_{i}$, is the linguistic label associated with this node function. In other words, $O_{i}^{1}$ is the membership function of $A_{i}$, and it specifies the degree to which the given $x$ satisfies the quantifier $A_{i}$.

2. Layer 2, the product layer: Every node in this layer is a fixed node labelled $\prod$ which multiplies the incoming signals and sends the product out. For instance,

$$
O_{i}^{2}=w_{i}=\mu_{A_{i}}(x) \times \mu_{B_{i}}(y), \quad i=1,2
$$

Each node output represents the firing strength of a rule. The output $w_{i}$ is the weight function of the next layer. 
3. Layer 3, the normalised layer: Every node in this layer is a fixed node labelled $N$. Its functions is to normalise the weight function in the following process:

$$
O_{i}^{3}=\bar{w}_{i}=\frac{w_{i}}{w_{1}+w_{2}}, \quad i=1,2
$$

4. Layer 4, the de-fuzzy layer: Every node $i$ in this layer is a adaptable node with a node function. The output equation of this layer is the Takagi-Sugeno type output $\bar{w}_{i}\left(p_{i} m+q_{i} n+r_{i}\right)$, where $p_{i}, q_{i}$ and $r_{i}$ denote the linear parameters or so-called consequent parameters of the node. The de-fuzzy relationship between the input and output of this layer can be defined as follows:

$$
\text { (22) } \quad O_{i}^{4}=\bar{w}_{i} f_{i}=\bar{w}_{i}\left(p_{i} m+q_{i} n+r_{i}\right), \quad i=1,2
$$

5. Layer 5, the total output layer: The single node in this layer is a fixed node labelled $\Sigma$ that computes the overall output as the summation of all incoming signals as

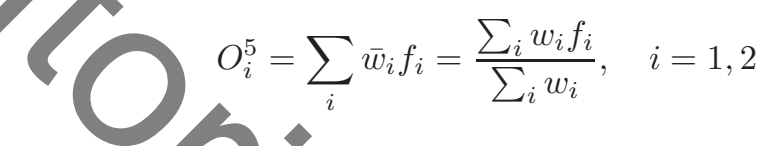

where $O_{i}^{5}$ denotes the layer 5 output.

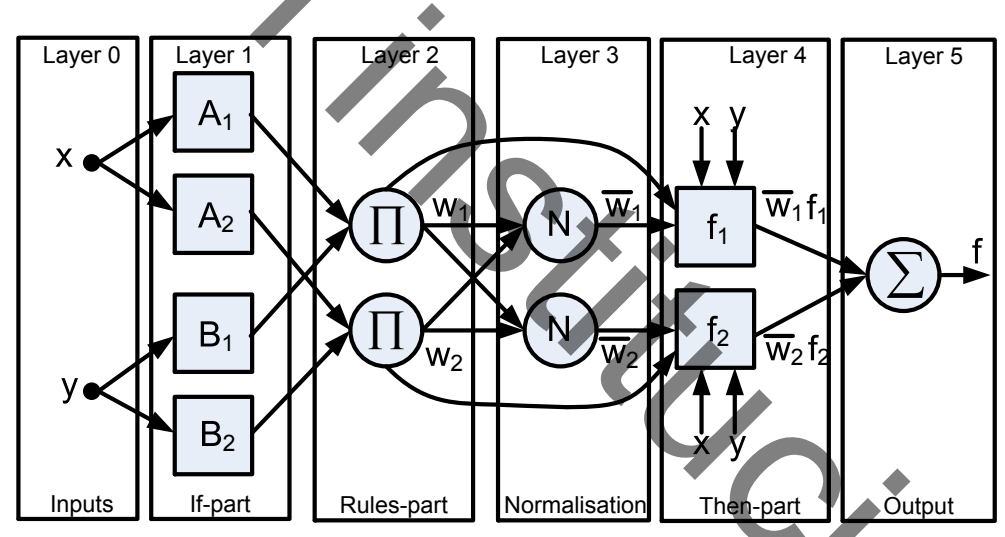

Figure 5 ANFIS structure defined with two inputs $x$ and $y$ and one output $z$. The nodes represented by squares are adaptable (their parameters are adjustable) whereas the others are fixed.

\subsubsection{Genetic Algorithms}

Genetic Algorithms (GAs) are search algorithms based on the mechanics of natural se lection and natural genetics, invented by Holland (1975), which can find the global optimal solution in complex multidimensional search spaces. A population of strings, representing solutions to a specified problem, is maintained by the GA. The GA then iteratively creates new populations from the old by ranking the strings and interbreeding the fittest to create new strings. So in each generation, the GA creates a set of strings from the previous ones, occasionally adding random new data to keep the population from stagnating. The end result is a search strategy that is tailored for vast, complex, multimodal search spaces. GAs are a form of randomised search, in that the way in which strings are chosen and combined 
is a stochastic process. This is a radically different approach to the problem solving methods used by more traditional algorithms, which tend to be more deterministic in nature such as the gradient methods. However, although GA is an effective optimisation algorithm, it usually takes a long time to find an optimal solution due to its slow convergence speed (Cus and Balic, 2003).

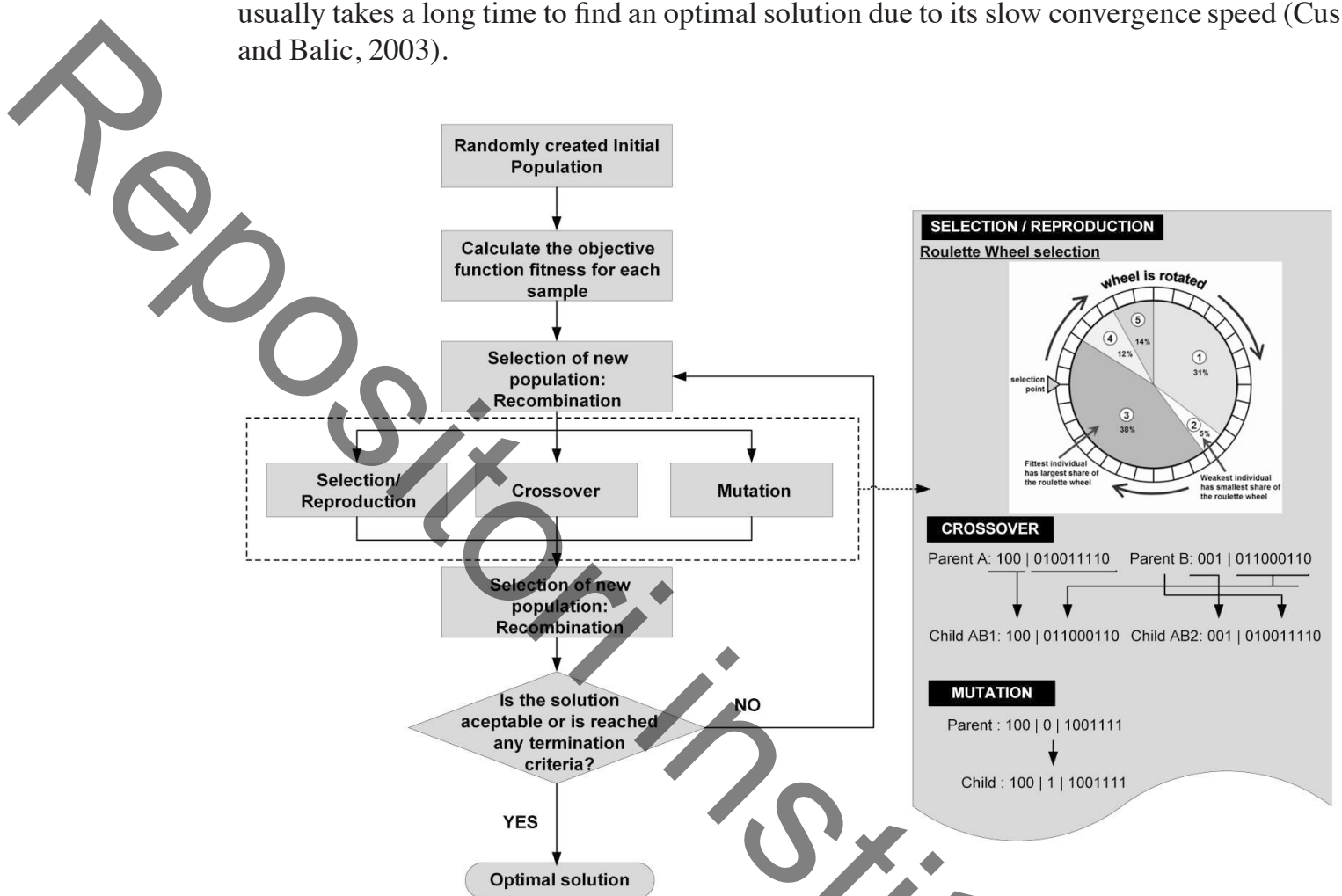

Figure 6 Flowchart of a basic genetic algorithm. The recombination step to create a new population is conducted by three genetic operators: reproduction/selection, crossover and mutation.

Figure 6 shows the iterative cycle of a basic genetic algorithm (adapted from Cus and Balic (2003)). Firstly, an initial population of strings is created. There two ways of forming this initial population. The first consists of using randomly produced solutions created by a random number generator, for example. This method is preferred for problems about which no a priori knowledge exists or for assessing the performance of an algorithm. The second method employs a priori knowledge about the given optimisation problem. Using this knowledge, a set of requirements is obtained and solutions that satisfy those requirements are collected to form an initial population. In this case, the GA starts the optimisation with a set of approximately known solutions, and therefore convergence to an optimal solution can take less time than with the previous method (Wang and Kusiak, 2000). The process then iteratively selects individuals from the population that undergo some form of transformation via the recombination step to create a new population. The new population is then tested to see if it fulfills some stopping criteria. If it does, then the process halts, otherwise another iteration is performed.

The recombination step in GAs is inspired by the natural evolution process, and it is basically conducted by three genetic operators: reproduction/selection, crossover and mutation. The main characteristics of each operator are defined as follows (Wang and $\mathrm{Ku}$ siak, 2000): 
- Reproduction/Selection: The aim of the selection procedure is to reproduce more of individuals whose fitness values are higher than those whose fitness values are low. The selection procedure has a significant influence on driving the search toward a promising area and finding good solutions in a short time. However, the diversity of the population must be maintained to avoid premature convergence and to reach the global optimal solution. There are many different types of reproduction operators such as roulette wheel selection, tournament selection, truncation selection, linear ranking selection and exponential ranking selection (Cus and Balic, 2003).

- Crossover: This operation is considered the one that makes the GA different from other algorithms, such as dynamic programming. It is used to create two new individuals (children) from two existing individuals (parents) picked from the current population by the selection operation. There are several ways of doing this. Some common crossover operations are one-point crossover, two-point crossover, cycle crossover, and uniform crossover. One-point crossover is the simplest crossover operation. Two individuals are randomly selected as parents from the pool of individuals formed by the selection procedure and cut at a randomly selected point. The tails, which are the parts after the cutting point, are swapped and two new individuals (children) are produced. An example of one-point crossover is shown in Figure 6.

- Mutation: In this procedure, all individuals in the population are checked bit by bit and the bit values are randomlly reversed according to a specified rate. Unlike crossover, this is a monadic operation. That is, a child string is produced from a single parent string. The mutation operator forces the algorithm to search new areas. Eventually, it helps the GA to avoid premature convergence and find the global optimal solution. An example is given in Figure 6, The mutation operation is controlled by the mutation rate.

The termination of GA could be done simply by counting if some prescribed number of steps is reached, or by testing, if a termination criterion is fulfilled. An autonomous stopping could be done by fitness function convergence testing of by homogeneity checking of an entire population. If fitness function reaches globalor some of local optima, then all strings, because of preferring the best solution, tend to be equivalent. Inthis case, a high degree of homogeneity could stop the procedure, or adapt GA parameters in order to move searching of the solution to other areas of local optima as candidates for the global one (Jain and Martin, 1998).

\subsection{Design of Experiments}

A full factorial $2^{3}$ design of experiments with two factors and three levels per factor was conducted to model the machining process and determine the optimal cutting parameters. The factors considered in the experimentation were the feed per tooth $\left(f_{z}\right)$ and the cutting speed $\left(V_{c}\right)$. The radial depth of cut $\left(a_{e}\right)$ was considered constant, with a value of 31.25 $m m$ to maximise the material removal rate and keep the cutting process in a steady-state. The axial depth of cut $\left(a_{p}\right)$ was defined as constant $(0.4 \mathrm{~mm})$ since the machining operation studied was a finishing operation. For each experiment, the face-milling operation was carried out until the cutting tool edge was worn (flank wear $-V_{b}$ - higher than $0.3 \mathrm{~mm}$, usual value for finishing operations (ISO, 1989)) or the surface roughness was outside specifica-

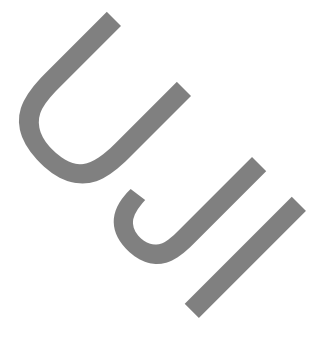


tions $(R a \geq 0.2 \mu m)$. Table 2 shows the cutting conditions analysed and the experimental results.

Table 2 Design of Experiments and experimental results.

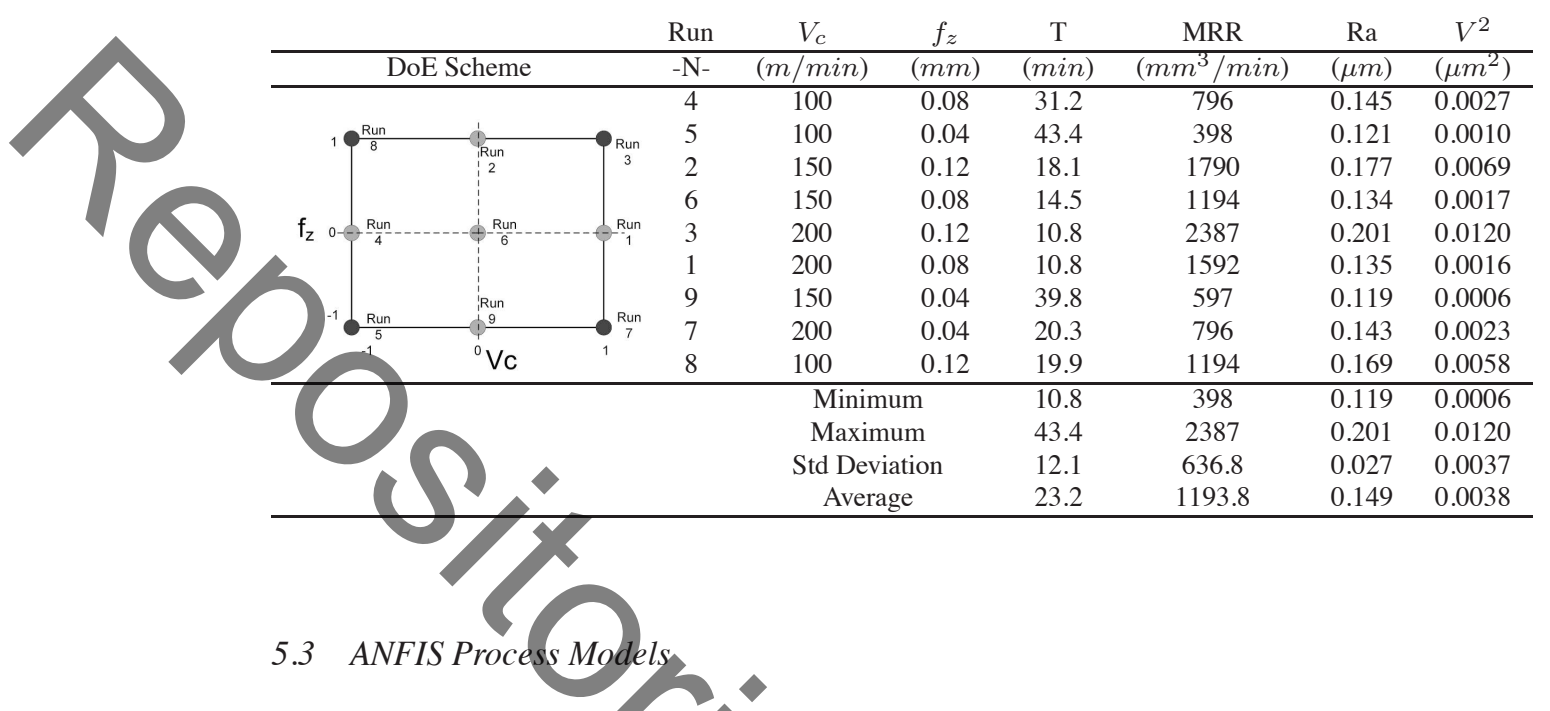

After experimentation, the experimental data was used to model the process through Adaptive Neuro-Fuzzy Inference Systems (ANFIS). Due to the few experimental data, the ANFIS models were initiated with two membership functions per each variable, representing the low and high variable value. The chosen membership function was the symmetric gaussian function which is defined by two parameters: the mean and the variance. As there are two variables ( $V_{c}$ and $f_{z}$ ) with two possible membership functions (low and high), there are four possible rules per each output variable. Therefore, to model each process output variable, ANFIS defines four rules according to the combinations of variables and membership functions. The output of each rule was defined as limear, so each output is composed of three coefficients in the form of $a V_{c}+b f_{z}+c$. For each output variable, four rules define the ANFIS model as follows:

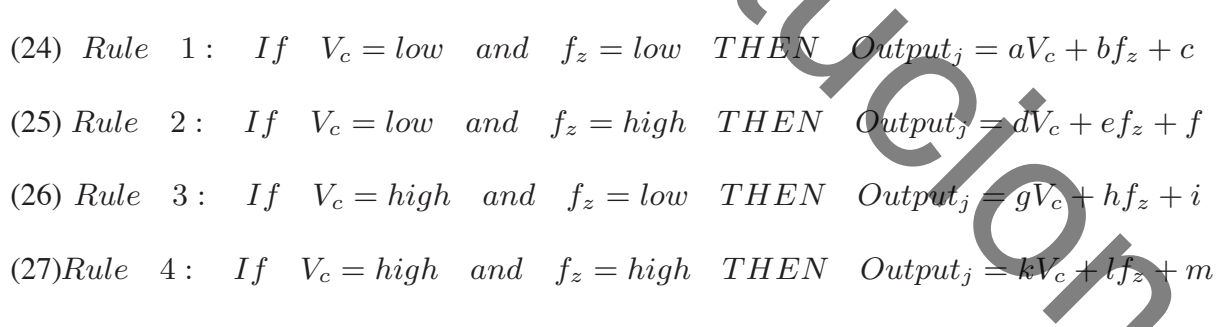

where Output ${ }_{j}$ can be the surface roughness, the cutting-tool wear state, the cutting-tool life or the quality loss. The membership functions and rules were adapted to fit the experimental data through a training procedure. The training was based on the hybrid optimisation procedure which is a combination of least-squares and backpropagation gradient descent method and the training was stopped at 150 epochs. After training, each ANFIS process model learnt the rules and membership functions which best describe the process. All rules learnt were analysed by the expert machinist, in order to verify the process knowledge extracted by the soft computing technique. Although most of the rules learnt were expected by the machinist, some of them were outside the expert machinist reasoning. With these unexpected rules, the machinist improved his process knowledge. Table 3 shows the main characteristics of the ANFIS models learnt. Table 4 shows the final ANFIS process models,

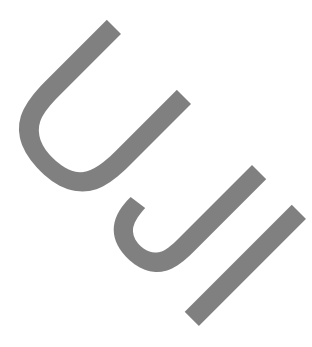


with the initial and final membership function for each variable, the rules learnt and the surface response. Table 5 shows the rules learnt after training each ANFIS model, with its physical explanation and the machinist's opinion.

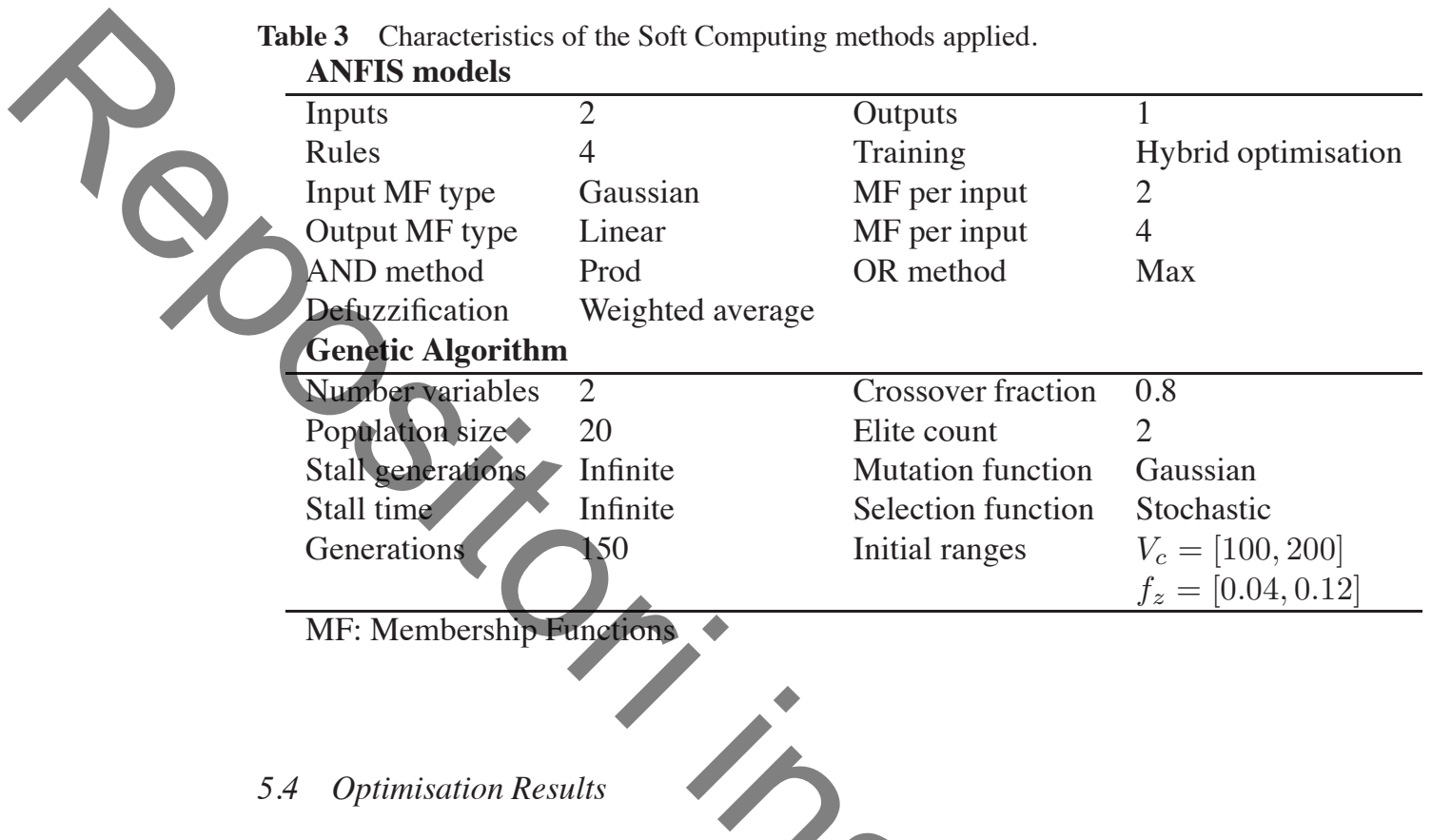

After modelling the machining process, the final desirability function can be defined as a function of $V_{c}$ and $f_{z}$. The optimal cutting parameter to maximise the overall desirability can be obtained through an optimisation algorithm. A genetic algorithm with the main characteristics shown in Table 3 was applied for cutting parameter optimisation. After 150 generations, the optimal cutting parameters were $V_{c} \Rightarrow 165 \mathrm{~m} / \mathrm{min}$ and $f_{z}=0.11$ $m m$, with an overall desirability function of 0.512 . In order to test experimentally the overall desirability function at the optimal cutting parameters, an experimental run with these cutting conditions was conducted, and a final desirability value of 0.536 was obtained. Therefore, the theoretical results estimated a desirability increase of $10.1 \%$, from 0.465 at $V_{c}=200 \mathrm{~m} / \mathrm{min}$ and $f_{z}=0.08 \mathrm{~mm}$ (machinist's approach) to 0.512 (soft computing approach). However, the experimental results showed an increase of the overall desirability function from 0.505 (machinist's approach) to 0.536 (soft computing approaeh), which means an increase of $6.1 \%$. The prediction error on the overall desirability function from $10.1 \%$ to $6.1 \%$ could be explained due to modelling errors, especially in modelling surface roughness and loss quality, which could be more complex than the estimated on

\section{Conclusions}

In this paper, a comparison between traditional cutting parameter optimisation using expert machinist knowledge and an experimental optimisation procedure based on Soft Computing methods has been reported. The presented experimental optimisation procedure has been composed of two steps. First, the modelling of the process variables of interest such as material removal rate, surface roughness, cutting tool life and loss quality of part accuracy 
Table 4 ANFIS process models for surface roughness (Ra), Tool-life ( $\mathrm{T}$ ), material removal rate (MRR) and loss quality (W)

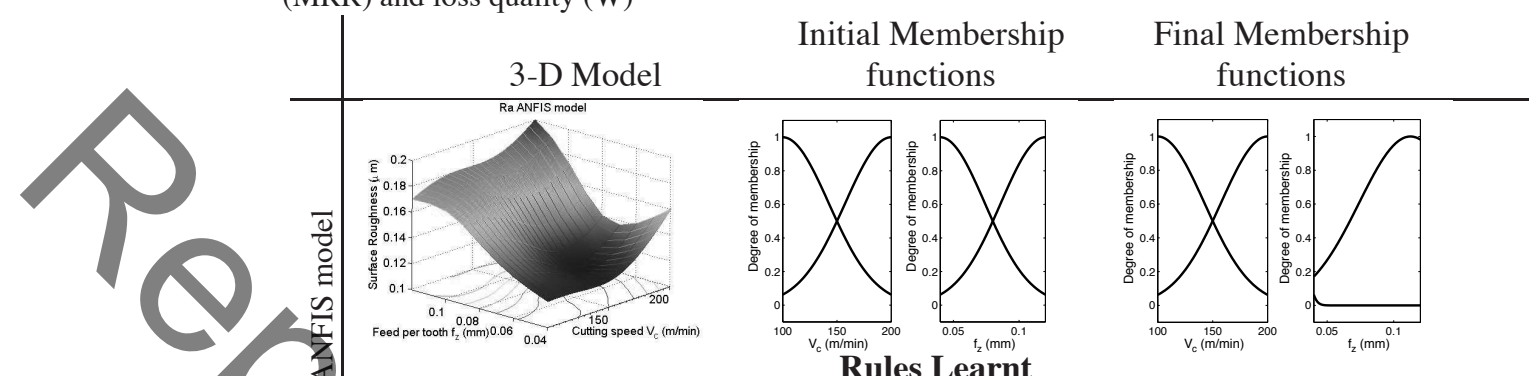

Rules Learnt

$\operatorname{IF}\left(V_{c}\right.$ is low) and $\left(f_{z}\right.$ is low) THEN $R a=5.610^{-4} V_{c}+0.0024 f_{z}+0.06$

$\operatorname{IF}\left(V_{c}\right.$ is low) and $\left(f_{z}\right.$ is high) THEN $R a=-6.710^{-5} V_{c}+0.51 f_{z}+0.11$

$\operatorname{IF}\left(V_{c}\right.$ is high) and $\left(f_{z}\right.$ is low) THEN $R a=1.8710^{-3} V_{c}-0.001 f_{z}-0.027$
$\operatorname{IF}\left(V_{c}\right.$ is high) and $\left(f_{z}\right.$ is high) THEN $R a=2.510^{-4} V_{c}+1.7 f_{z}-0.051$

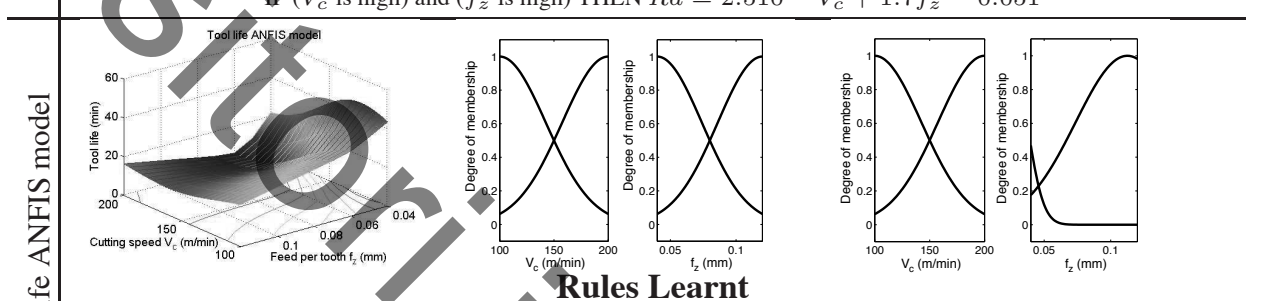

Rules Learnt

$\operatorname{IF}\left(V_{c}\right.$ is low) and $\left(f_{z}\right.$ is low) THEN $T=0.6 V_{c}-0.49 f_{z}-12.57$

$\operatorname{IF}\left(V_{c}\right.$ is low) and $\left(f_{z}\right.$ is high) THEN $T=0.022 V_{c}-223.9 f_{z}+47.38$

$\operatorname{IF}\left(V_{c}\right.$ is high $)$ and $\left(f_{z}\right.$ is low $)$ THEN $T=0.074 V_{c}+0.223 f_{z}+5.68$

$\operatorname{IF}\left(V_{c}\right.$ is high $)$ and $\left(f_{z}\right.$ is high) THEN T $=0.107 V_{c}+95.7 f_{z}-21.4$

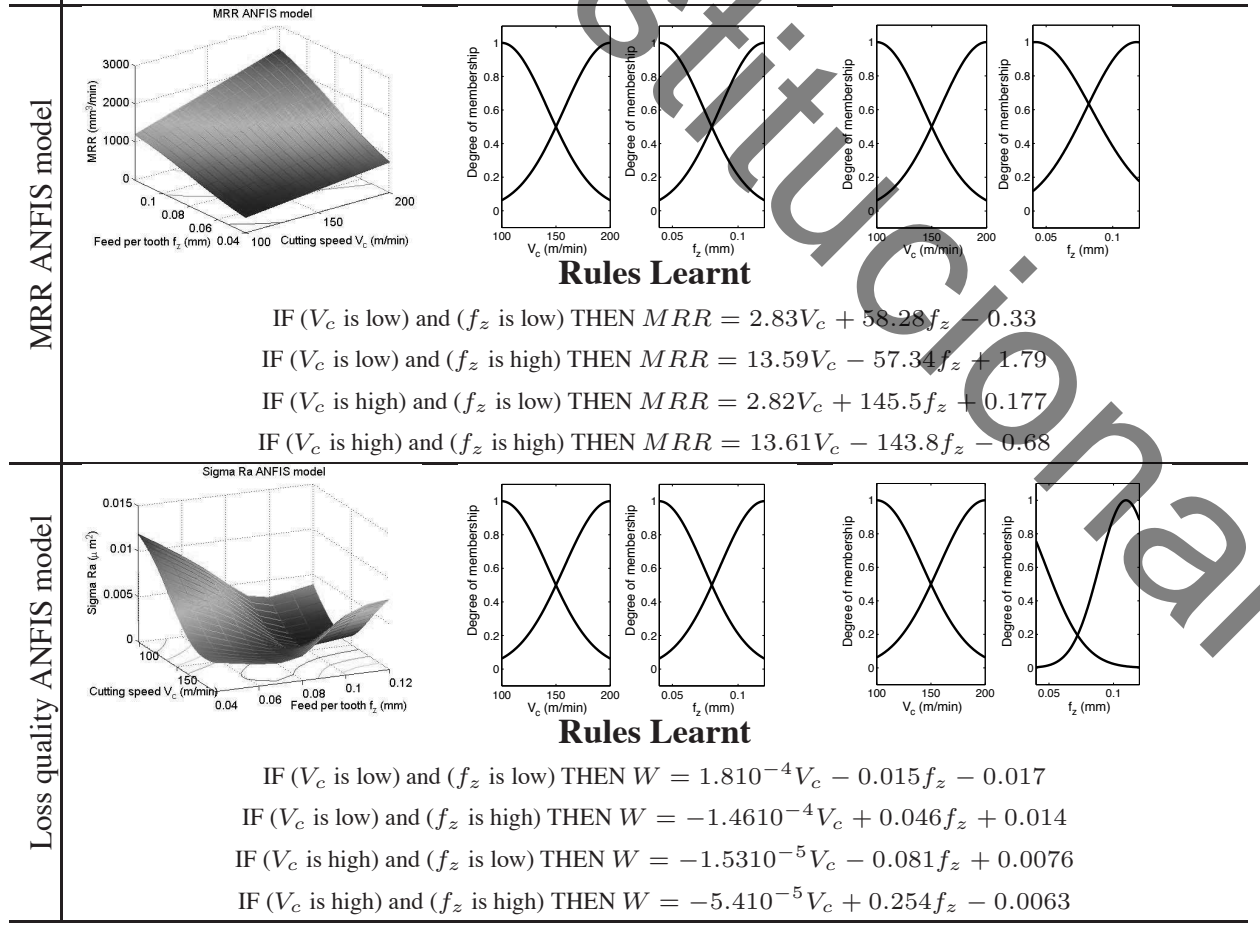




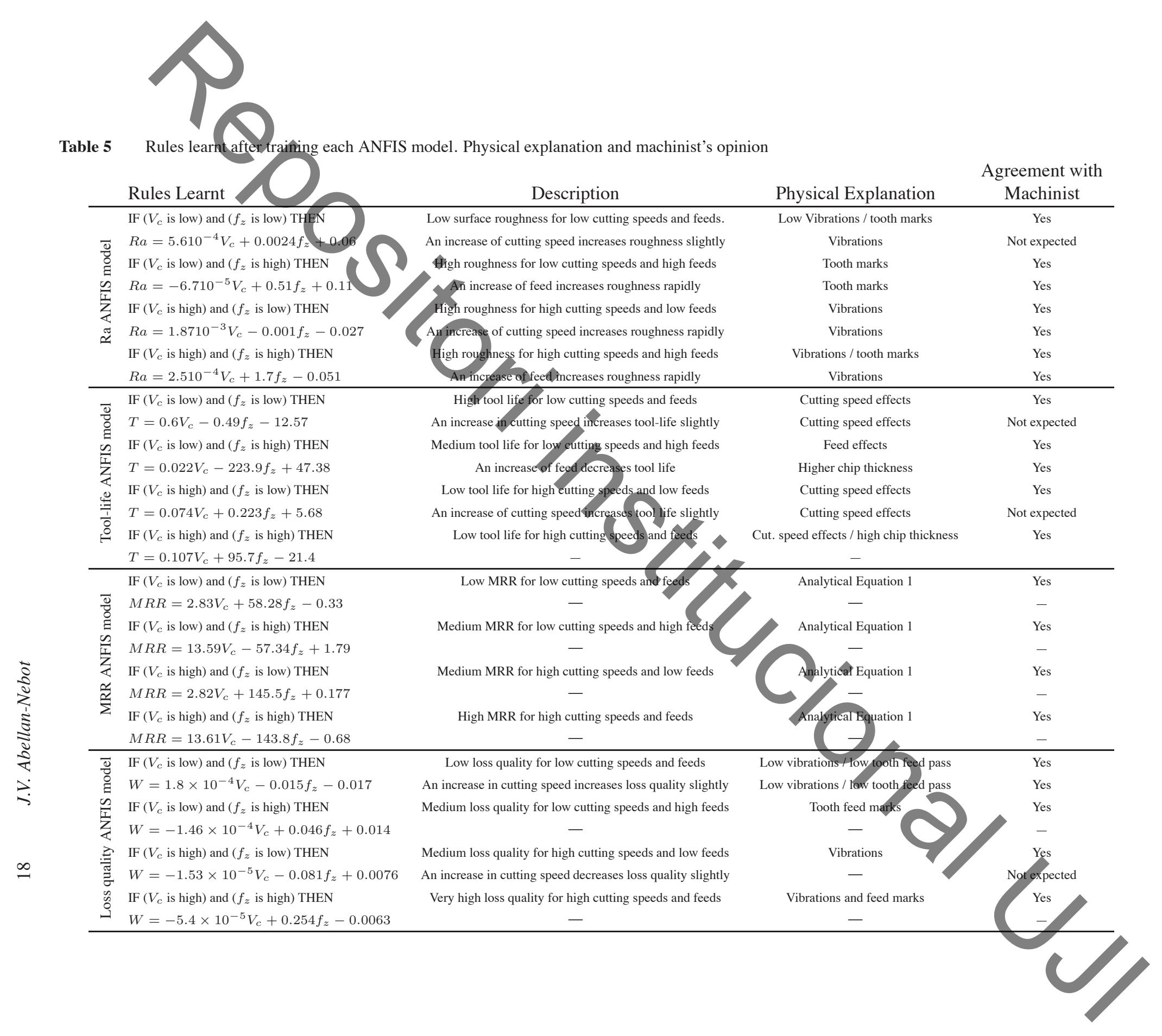


have been conducted by Adaptive Neuro-fuzzy Inference Systems. This soft computing approach models each process variable by rules extracted from the experimental data which can be interpreted and verified by the expert machinist in order to have a better understanding of the process. As a second step, it was defined a multi-objective function using the desirability function approach and a Genetic Algorithm was applied to optimise the overall desirability function and find the optimal cutting parameters which best define the trade-off among production rate, cutting costs and part accuracy. The theoretical results predicted an increase on the multi-objective function of $10.1 \%$ by the application of the experimental cutting parameter approach presented in the paper instead of the parameter selection by the expert machinist. After a validation run, the increase on the multi-objective function was proved to be $6.1 \%$. The error between theoretical and experimental results were assumed be due to modelling errors, especially in the surface roughness and loss quality models.

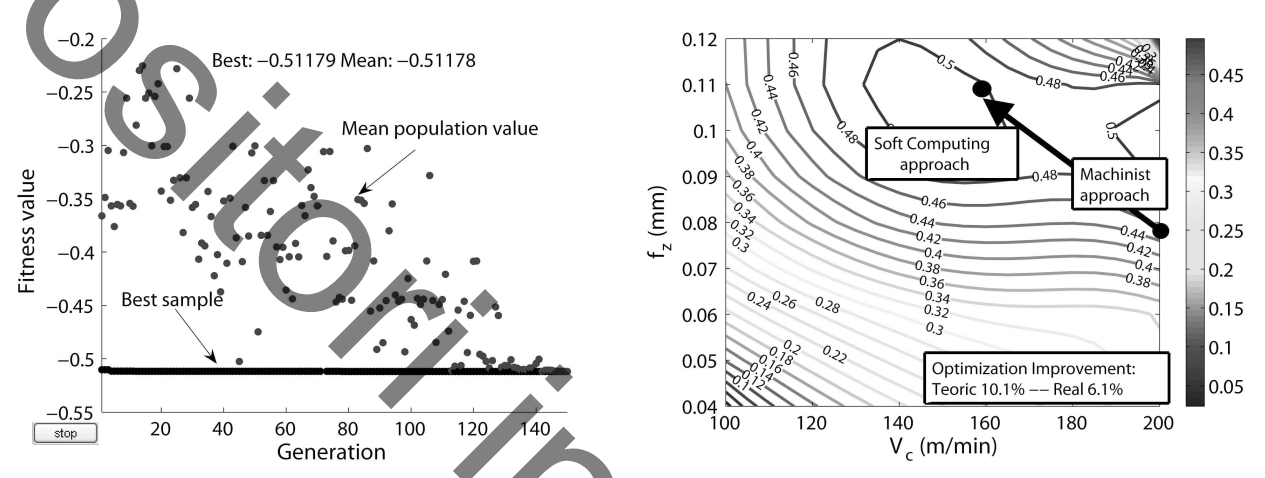

(a)

(b)

Figure 7 (a) Mean population value and best sample at each generation during the optimisation by GA. (b) Comparison between the overall desirability function obtained by using the expert machinist knowledge and the Soft Computing method proposed.

\section{References}

Chiang, S. T., Liu, D. I., Lee, A. C. and Chieng, W. H. (1995) Adaptiye-control optimization in end milling using neural networks', International Journal of Machine Tools and Manufacture, Vol. 35, No. 4, pp. 637-660.

Chien, W. T. and Chou, C. Y. (2001) 'The predictive model for machinability of 304 stainless steel', Journal of Materials Processing Technology, Vol. 118, No. 1-3, pp. 442-447.

Cus, F. and Balic, J. (2003) 'Optimization of cutting process by ga approach', Robotics and Computer-Integrated Manufacturing, Vol. 19, No. 1-2, pp. 113-121.

Derringer, G. and Suich, R. (1980) 'Simultaneous-optimization of several response variables', Journal of Quality Technology, Vol. 12, No. 4, pp. 214-219.

Ghani, J. A., Choudhury, I. A. and Hassan, H. H. (2004) 'Application of taguchi method in the optimization of end milling parameters', Journal of Materials Processing Technology, Vol. 145, No. 1, pp. 84-92.

Holland, J. (1975) Adaptation in natural and artificial systems, Ann Arbor, USA: The University of Michigan Press. 
Ip, R. W. L., Lau, H. C. W. and Chan, F. T. S. (2003) 'An economical sculptured surface machining approach using fuzzy models and ball-nosed cutters', Journal of Materials Processing Technology, Vol. 138, pp. 579-585.

ISO (1989) 'Tool life test in milling, part 1: Face milling', International Standard Organization (ISO).

Jain,L.C. and Martin, N. M. (1998) Fusion of Neural Networks, Fuzzy Systems and Genetic Algorithms: Industrial Applications, Florida, CRC Press LLC edn.

Jang, J. S. R. (1993) 'Anfis - adaptive-network-based fuzzy inference system', Ieee Transactions on Systems Man and Cybernetics, Vol. 23, No. 3, pp. 665-685.

Juan, H., Yu, S. F. and Lee, B. Y. (2003) 'The optimal cutting-parameter selection of production cost in hsm for skd61 tool steels', International Journal of Machine Tools and Manufacture, Vol. 43, No. 7, pp. 679-686.

Liu, Y. M. and Wang, C. J. (1999a) 'Neural network based adaptive control and optimisation in the milling process', International Journal of Advanced Manufacturing Technology, Vol. 15, No. 11, pp 791-795.

Liu, Y. M., Zuo, L. and Wang, C. J. (1999b) 'Intelligent adaptive control in milling processes', International Journal of Computer Integrated Manufacturing, Vol. 12, No. 5, pp. 453-460.

Mukherjee, I. and Ray, P. K. (2006) 'A review of optimization techniques in metal cutting processes', Computers and Industrial Engineering, Vol. 50, No. 1-2, pp. 15-34.

NIST/SEMATECH (2006) 'Nist/sematech e-handbook of statistical methods', Electronic Citation.

Siller, H. R., Vila, C., Rodriguez, C. A. and Abellan, J. V. (2008) 'Study of face milling of hardened aisi d3 steel with a special design of carbide tool', International Journal of Advanced Manufacturing Technology.

Suresh, P. V. S., Rao, P. V. and Deshmukh, S. G. (2002) A genetic algorithmic approach for optimization of surface roughness prediction moder, International Journal of Machine Tools and Manufacture, Vol. 42, No. 6, pp. 675-680.

Trent, E. M. and Wright, P. K. (2000) Metal Cutting, ButterworthHeinemann, fourth edition edn.

Wang, J. and Kusiak, A. (2000) Computational Intelligence in Manufacturing Handbook.

Zhang, J. Z., Chen, J. C. and Kirby, E. D. (2007) 'Surface roughness optimization in an end-milling operation using the taguchi design method', Journal of Materials Processing Technology, Vol. 184, No. 1-3, pp. 233-239.

Zuperl, U. and Cus, F. (2003) 'Optimization of cutting conditions during cutting by using neural networks', Robotics and Computer-Integrated Manufacturing, Vol. 19, No.1-2, pp. 189-199. 